\title{
Optimal Combinatorial Functions Comparing Multiprocess Allocation Performance in Multiprocessor Systems
}

\author{
Håkan Lennerstad* \\ Dept. of Mathematics \\ Univ. of Karlskrona/Ronneby \\ S-371 79 Karlskrona
}

\author{
Lars Lundberg* \\ Dept. of Computer Science \\ Univ. of Karlskrona/Ronneby \\ S-372 25 Ronneby
}

\section{Abstract}

For the execution of an arbitrary parallel program $P$, consisting of a set of processes, we consider two alternative multiprocessors.

The first multiprocessor has $q$ processors and allocates parallel programs dynamically, i.e. processes may be reallocated from one processor to another. The second employs cluster allocation with $k$ clusters and $u$ processors in each cluster - here processes may be reallocated within a cluster only. Let $T_{d}(P, q)$ and $T_{c}(P, k, u)$ be execution times for the parallel program $P$ with optimal allocations. We derive a formula for the program independent performance function

$$
G(k, u, q)=\sup _{\text {all parallel programs } P} \frac{T_{c}(P, k, u)}{T_{d}(P, q)} .
$$

Hence, with optimal allocations, the execution of $P$ can never take more than a factor $G(k, u, q)$ longer time with the second multiprocessor than with the first, and there exist programs showing that the bound is sharp.

The supremum is taken over all parallel programs consisting of any number of processes. Any interprocess dependency structure is allowed for the parallel programs, except deadlock. Overhead for synchronization and reallocation is neglected only.

We further present optimal formulas which exploits a priori knowledge of the class of parallel programs intended for the multiprocessor, thus resulting in sharper optimal bounds. The function $g(n, k, u, q)$ is the above maximum taken over all parallel programs consisting of $n$ processes. The function $s(n, v, k, u)$ is the same maximum, with $q=n$, taken over all parallel programs of $n$ processes which has a degree of parallelism characterized by a certain parallel profile vector $v=\left(v_{1}, \ldots, v_{n}\right)$.

\footnotetext{
*Both authors were supported in part by the Blekinge Research Foundation.
} 
The functions can be used in various ways to obtain optimal performance bounds, aiding in multiprocessor architecture decisions. An immediate application is the evaluation of heuristic allocation algorithms.

It is well known that the problems of finding the corresponding optimal allocations are NP-complete. We thus in effect present a methodology to obtain optimal control of NP-complete scheduling problems.

\section{Problem definition and results}

This report establishes optimal worst case bounds comparing the execution time for a program on two multiprocessors. The multiprocessors have identical processors but different organization and different processor quantity.

The multiprocessors execute parallel programs $P$ which may have any set of runtimes and any possible structure of interprocess dependency, only excepting deadlock. The parallel program model under consideration is general. A parallel program consists of a set of processes, some of which may run in parallel, and a set of dependencies between the processes, called syncronization, in the sense that one process, at a specific point in the process, may not execute unless another process has reached a certain point. We have no limitations of the occurence of such dependencies in a program, other that the program is required to be executable. For example, some programs include time consuming transferring of data between subprocesses. Then the transferring itself can be viewed as a part of the process, while the dependency is realized as synchronization signals of no time duration at several points during the transferring.

In cluster allocation the processors are organized in $k$ groups, the clusters, where each cluster contains $u$ processors. Here we thus have in total $k u$ processors. Once a process is allocated to a processor in a specific cluster it can only be executed on a processor in this cluster to completion. It may be transferred any time, but only to a processor in the same cluster. The cost of all transferring of processes is neglected. If a process is put into a waiting state, it will thus later be restarted on a processor in the same cluster.

Dynamic and static allocations are both special cases of cluster allocation. Dynamic allocation represents the case of having all processors in one cluster, $k=1$, hence the processes may be transferred between all processors without limits. In static allocation each cluster has one single processor, $u=1$, thus a process may never be transferred from the processor where it was initiated.

For a parallel program $P$, there is a certain number of different cluster allocations. This number is usually very large, however certainly finite. The execution time of each alloation is affected by the order in which the processes allocated to the same cluster are executed. However, each of the allocations of the program has a well defined minimal execution time. We are interested in the minimal execution times for two multiprocessor organizations. Therefore, we only need to consider the minimal execution time for each allocation.

Since we now have a well defined execution time for each allocation, the set of execution times of the program $P$ for all cluster allocations is finite, so it has a minimum. An 
allocation, which results in an execution time shorter than or equal to that of any other alloation is called an optimal allocation scheme. It is well known that it is a NP-complete problem to find an optimal cluster allocation.

For any fixed parallel program $P$, we compare the minimal execution time of $P$ for two multiprocessors.

The first multiprocessor has $q$ processors and allocates parallel programs dynamically. For a parallel program $P$, we denote the execution time for $P$ with optimal dynamic allocation by $T_{d}(P, q)$, i.e. with a dynamic allocation such that there is no other dynamic allocation for which the execution time of $P$ is shorter.

The second multiprocessor performs cluster allocation with $k$ clusters and $u$ processors in each cluster. Let $T_{c}(P, k, u)$ denote the execution time for the parallel program $P$ with optimal cluster allocation.

Overhead for synchronization and reallocation is neglected throughout the report. In section 4 the allocation problem is defined in detail.

The allocation problem will be formulated as a mathematical optimization problem about so called 0,1-matrices, i.e. matrices where all entries are 0 or 1 . The extremal matrices, corresponding to extremal parallel programs, are characterized. This makes it possible to establish a formula for the performance function $g$ :

$$
g(n, k, u, q)=\underset{\text { all }}{\max \text {-programs } P} \frac{T_{c}(P, k, u)}{T_{d}(P, q)} .
$$

Here $n$-programs denotes parallel programs with $n$ processes. The formula is presented in section 6 .

The degree of parallelism for a program $P$ can be characterized by a vector $v=$ $\left(v_{1}, \ldots, v_{n}\right)$. Here $v_{i}$ is the percentage of the total execution time where exactly $i$ processes execute simultaneously if each process is allocated to a separate processor. From the function $g$ we derive a formula for the performance function

$$
s(n, v, k, u)=\max _{P} \frac{T_{c}(P, k, u)}{T_{d}(P, n)},
$$

where the maximum is taken over all parallel programs $P$ of $n$ processes which has the parallel profile vector $v$.

The function $g(n, k, u, q)$ is proven to be incresing in the variable $n$, a fact which can be used to compute a formula for the process independent performance function

$$
G(k, u, q)=\sup _{\text {all parallel programs } P} \frac{T_{c}(P, k, u)}{T_{d}(P, q)}=\lim _{n \rightarrow \infty} g(n, k, u, q) .
$$

This performance function is applicable for a multiprocessor intended for any parallel program. No prior knowledge of the parallel programs can be used to extract further information from the function $G(k, u, q)$. On the other hand the functions $g(n, k, u, q)$ and $s(n, v, k, u)$ are useful when there is prior knowledge of certain kinds about the parallel programs.

Note that beside the allocations, the performance functions themselves are optimal, while representing bounds which cannot be improved. The term "optimal" thus appears in two senses. 


\section{Previous results and applications}

The present report extends the results in [2] and [6]. Here the the formula for the function $g$ in the case $u=1, k=q$ is treated only, representing static versus dynamic allocation on the same multiprocessor.

The mathematics of the subject is focused in this report. It can be viewed as the theoretical fundament for the reports [4], [7], [8] and [10], which treat different applications from a computer science point of view.

The report [9] provides an application not covered by the present report. Here a test execution of the program can be used to provide sharper bounds. The results involve linear programming where values of the function $G$ occur as cofficients.

Furthermore, the basic method presented here has proved to be useful in other contexts. In the report [11], the efficiency of cache memories for single processors is studied. Here optimal bounds comparing more flexible with less flexible cache memory organization alternatives are derived, the final part of the argument is similar to that of the present report. [5] is a survey article of the results in [11].

Since the problems of finding optimal allocations are NP-complete, general and preferably optimal results are needed already for a medium number of processors and processes in order to choose multiprocessor architecture which opimizes performance. Other than the reports [2] to [14], the only general results concerning allocation strategies of parallel programs appear to be the results by R. L. Graham [1]. The overhead for process reallocation and synchronization is neglected also in that work. So called self-scheduling algorithms are considered. This term is used for dynamic allocation algorithms where, when a processor becomes idle and there are waiting executable processes, one of these is immediately allocated to this processor. It is established in [1] that the execution time for a program allocated with a self-scheduling algorithm is never higher than two times the execution time with optimal dynamic allocation.

The process allocation scheme is of major importance for the performance of a multiprocessor, thus an immediate area of applications for the present results is multiprocessor design. The results can further be used to evaluate the efficiency of cluster allocation algorithms versus the best possible algorithm, i.e. the algorithm which finds the optimal cluster allocation. One difficulty in this application which concerns the functions $G$ and $g$ is that this execution time is compared to the execution time with optimal dynamic allocation, which is unknown and NP-hard. The function $s$ compares the optimal cluster allocation with the case of having each process allocated to a processor on its own, hence the optimal allocation is obvious and the difficulty does not arise in this case.

The most general result in this report is clearly the one represented by the function $G$. Here we have no demands on the program. The function $g$ is restricted to programs with $n$ processes, while the function $s$ concerns programs with $n$ processes and a certain parallel profile. It is expected that the techniques presented here can be extended to take advantage of further kinds of program specifics, thus improving the bounds by keeping away from those programs which maximize the ratio studied in this work. In effect we present a methodology to obtain optimal control of NP-complete scheduling problems.

One example of a multiprocess architecture feature which immediately follows from the 
function $G(k, u, q)$ is the number of extra processors which would compensate for a more static allocation, i.e. the trade off between multiprocess power and allocation efficiency. The concluding graphics section shows plots of this and many other ways to exploit the performance functions for multiprocessor architecture purposes.

As a mathematical result it is applicable for scheduling problems in other contexts. For example, the construction of a house is clearly a multiprocess with subprocesses which are in some respects dependent and in some respects independent. Here the processors are not as general as computers in the sense that some processes probably cannot efficiently be allocated to all processors. Humans are usually specialists. Within automation and robotics scheduling problems are frequent, and here the skills of the processors can often be clearly defined.

We next give an overview of the report.

In the following section the allocation problem is described and analyzed in detail, and transformed to a mathematical problem. In section 5 we give a full formulation of the mathematical problem and introduce necessary notation. In section 6 the formulas for the program dependent performance functions $g(n, k, u, q)$ and $s(n, v, u, q)$ are stated and proved. Basic properties of the function $g(n, k, u, q)$ are also established here. Section 7 deals with the general performance function $G(k, u, q)$. The report is concluded with a graphics section. The purpose of this section is twofold: to show the performance functions quantitatively and geometrically, and to suggest how the functions can be used to get optimal aid in multiprocessor design decisions.

\section{From programs to matrices}

A program $P$ consists of $n$ processes of possibly very different execution times. The processes are of course usually dependent of each other. One can expect dependencies of the type that process $i$ cannot execute further at the time point $t_{i}$ unless process $j$ has reached the time point $t_{j}$. When process $j$ has reached the time point $t_{j}$ it is said to execute a synchronizing signal to process $i$, restarting this process. Certainly there can be many synchronizing signals to a time point $t_{j}$, in which case all have to be executed before the process restarts. The execution time of synchronizing signals is neglected. Most parallel programs contain many synchronizing signals. In this report any set of synchronization signals is allowed, except those which include a deadlock.

Thus a parallel program $P$ of $n$ processes is defined by the total execution times of the $n$ processes and by the set of synchronizing signals.

Now consider a parallel program $P$. Assume that we have found an optimal dynamic allocation, with execution time $T_{d}(P, q)$. This optimal dynamic allocation will be kept fixed during the entire argument dealing with the program $P$ and its descendant $P^{\prime}$. Next we introduce a discretization of the time interval in subintervals $\left(t_{i}, t_{i+1}\right)$ of equal length, such that all synchronizing signals, process initiations and process terminations appear on the time points $t_{i}$, where $t_{i}=\frac{i}{m} T_{d}(P, q), i=0, \ldots, m$. Obviously all processes in the interval $\left(t_{i-1}, t_{i}\right)$ are completed before any part of the processes corresponding to 
the interval $\left(t_{i}, t_{i+1}\right)$ when using this allocation, since this is so without the discretization. Such a discretization is possible if all synchronizing signals and process terminations occur at rational time points, which we can assume. Observe that $m$ might be very large even if the program $P$ is small and has a simple structure.

For the sake of the function $s(n, v, k, u)$, let $v_{1}, \ldots, v_{n}$ be the parallel profile of the program $P$ : when having the processes allocated on separate processors, $i$ processors are active simultanouosly exactly the share $v_{i}$ of the total execution time of $P$. The numbers $v_{i}$ are assumed to be non-negative rational numbers such that $\sum_{i=1}^{n} v_{i}=1$. Then $m$ is necessarily a multiple of the smallest common divisor of the numbers $v_{1}, \ldots, v_{n}$. There are integers $x_{i}=m v_{i}$ so that exactly $x_{i}$ intervals have $i$ active processes.

Consequently, during a time interval $\left(t_{i}, t_{i+1}\right)$, no process of the program $P$ starts, and no process stops.

From the program $P$ and for the functions $G$ and $g$, we next construct another program $P^{\prime}$ by two changes of the program $P$ : we introduce new synchronizing signals and prolong certain processes. For the sake of the function $s(n, v, k, u)$ only one of these changes is done: new synchronization is introduced. Any prolonging of processes would destroy the specific parallel profile character, hence for this case the only difference between the programs $P$ and $P^{\prime}$ is the synchronization. At every time point $t_{i}$ we introduce all possible synchronization between the processes. This means that the synchronization now requires that all processes in the interval $\left(t_{i-1}, t_{i}\right)$ have to be completed before any part of the processes corresponding to the interval $\left(t_{i}, t_{i+1}\right)$, which will increase the execution time with most other allocations. Since the execution time of synchronizing signals is neglected this does not change the total execution time with the fixed optimal dynamic allocation, which is $T_{d}(P, q)$. Further, for the functions $G$ and $g$, all processors are made to be busy at all time intervals. This is achieved by, if necessary, prolonging some processes. However no process is prolonged beyond $T_{d}(P, q)$, hence $T_{d}(P, q)=T_{d}\left(P^{\prime}, q\right)$. It is of no importance that the prolonging of processes can be made in many ways; many programs can play the role of $P^{\prime}$ to a specific program $P$.

By the construction we thus have $T_{d}(P, q)=T_{d}\left(P^{\prime}, q\right)$. However, since introducing more synchronization and prolonging processes never shortens the execution time, for other allocations the execution time is either increased or unchanged. In particular, for optimal cluster allocation we therefore have $T_{c}(P, k, u) \leq T_{c}\left(P^{\prime}, k, u\right)$. Consequently,

$$
\frac{T_{c}(P, k, u)}{T_{d}(P, q)} \leq \frac{T_{c}\left(P^{\prime}, k, u\right)}{T_{d}\left(P^{\prime}, q\right)}
$$

Certainly there are programs $P$ which are left unchanged by the above transformation: programs such that $P=P^{\prime}$. Since these programs constitute a subset of the parallel programs we consider, we actually have

$$
\max _{P} \frac{T_{c}(P, k, u)}{T_{d}(P, q)}=\max _{P} \frac{T_{c}\left(P^{\prime}, k, u\right)}{T_{d}\left(P^{\prime}, q\right)} .
$$

Therefore, in order to calculate the maximum, only programs of the type $P^{\prime}$ need to be considered. 
We next represent a program $P^{\prime}$ by an $m \times n$ matrix of $0:$ s and 1:s only. Here each process is represented by a column, and each time period is represented by a row. The entry at the position $(i, j)$ of the matrix is 1 if the $j$ :th process is active between $t_{i-1}$ and $t_{i}$; if it is inactive the entry is 0 . Each row contains exactly $q 1: \mathrm{s}$, since each processor is constantly busy. In the sequel such a matrix is referred to as an $m, n, q$-type matrix. The main part of the report analyzes these matrices. For example we characterize the type of matrix which corresponds to the worst case. Because of the complete synchronization, each row has to be completed before the next row. The execution time of the program $P^{\prime}$ with optimal dynamic allocation in this time unit is $m$. For the formula for $s$ there is exactly $x_{i}$ rows which has $i 1: \mathrm{s}, \sum_{i=1}^{n} x_{i}=m$. The matrix for this case is composed by $n$ $x_{i}, n, i$-type matrices.

Our next objective is to compute the optimal cluster execution time of the program $P^{\prime}$.

To compute this we need to decide how the $n$ processes are to be allocated to the $k$ clusters. Since every process in this case is to be executed within one cluster only, the cluster allocation corresponds to a way of grouping the $n$ columns of the matrix together in $k$ sets, one set for each cluster. Assume $l$ processes are allocated to a specific cluster at a specific time inteval. Within a cluster there are $u$ processors and the processes are allocated dynamically. Since there is no synchronization within a time interval, the programs are independent and we will next see that the execution time is here $\max (l / u, 1)$. The execution time clearly cannot be lower than $l / u$. Further, the execution time cannot be lower than 1 since each process is considered to be completely synchronized to itself. That is, no part of a process can be executed in parallel with another part of the same process. In the case $l \geq u$ the limit $l / u$ is reached, which can be seen by dividing the execution time for each processor in $l$ parts of size $1 / u$ each. On the first processor each process is executed during exactly one of the intervals of size $1 / u$. On the second processor this execution scheme is permuted cyclically. On the $i$ :th processor, the same cyclic permutation is carried out $i$ times. With this execution scheme for the processors, the total execution time is $l / u$ and no process is executed in parallel with itself.

Because of the complete synchronization at the time points $t_{i}$, processing cannot proceed before completion of the slowest cluster. Hence, we obtain a maximum of $\max (l / u, 1)$ over the $k$ clusters. The execution time with cluster allocation in the time unit given by the interval length is the sum of these maximas. This is the optimal cluster execution time $T_{c}\left(P^{\prime}, k, u\right)$ if we have found an allocation of the $n$ columns together in $k$ sets which minimizes the cluster execution time.

If we change the time unit so that $T_{d}(P, q)=m$, then $T_{c}\left(P^{\prime}, k, u\right)$ is an integer, and will be denoted by $T\left(P^{\prime}, k, u\right)$.

In section 6 we establish a formula for the function $g(n, k, u, q)$ representing the worst case, i.e. for any program $P^{\prime}$ :

$$
\frac{T\left(P^{\prime}, k, u\right)}{T_{d}\left(P^{\prime}, q\right)} \leq g(n, k, u, q)=\max _{P} \frac{T_{c}(P, k, u)}{T_{d}(P, q)}=\max _{P} \frac{T(P, k, u)}{m} .
$$

From the function $g$ formulas for the performance functions $s$ and $G$ can be derived. 


\section{The matrix problem}

The operator $T$ with no index in the equation above belongs to the mathematical formulation. It is a function, to be defined, taking 0,1-matrices as arguments and having integers as values. $T(P, k, u)$ represents the execution time with optimal cluster allocation, having $k$ clusters with $u$ processors in each, while $m$ is the dynamic analogue with $q$ processors. For simplicity of notation we will frequently write $T(P)$ instead of $T(P, k, u)$.

In $T(P), P$ is an $m \times n$ matrix $P$ of $0: s$ and 1 :s only, such that each row has exactly $q 1$ :s, and thus $n-q 0: \mathrm{s}, 1 \leq q \leq n$. We say that a matrix $P$ of this type is of $m, n, q-$ type. Thus any program with $n$ processes which is executed with dynamic allocation on a processor with $q$ processors is represented by a $m, n, q$-type matrix $P$.

Consider an $m, n, q$-type matrix $P$ and a partition $A$ of the $n$ column vectors into $k$ sets. We will be mostly concerned with partitions where the sizes of the sets in the partition differ as little as possible. If $n / k$ is an integer $w$, every set in such a partition has $w$ members. Denote the integer part of $n / k$, the floor function, by $\lfloor n / k\rfloor$, and the smallest integer greater than or equal to $n / k$, the ceiling function, by $\lceil n / k\rceil$. If $n / k$ is not an integer, the sets in a partition where the sizes differ as little as possible have $\lfloor n / k\rfloor$ or $\lceil n / k\rceil$ members.

Denote the number of 1:s in cluster $l$ at row $j$ by $c(l, j)$. As described in the previous section, the execution time with cluster allocation using the partition $A$ is then

$$
T_{A}(P, k, u)=\sum_{j=1}^{m} \max _{l=1, \ldots, k}(c(l, j) / u, 1) .
$$

We want to choose the partition $A$ so that $T_{A}(P)$ is minimal. The execution time with optimal cluster allocation is thus

$$
T(P)=\min _{\text {all partitions } A} T_{A}(P) .
$$

We can now define the performance function $g(n, k, u, q)$ :

$$
g(n, k, u, q)=\max \left\{\frac{T(P, k, u)}{m} \text {, all } m, n, q-\text { type matrices } P\right\} .
$$

The function $g$ is here defined only for $k \leq n$ and $q \leq n$. The definition is extended to $k>n$ by $g(n, k, u, q)=1$, and to $q>n$ by $g(n, k, u, q)=g(n, k, u, n)=\max \left(\left\lceil\frac{n}{k}\right\rceil / u, 1\right)$. This is in accordance with the application since in these cases we have unnecessary clusters and unnecessary processors, respectively. That the extension to $k>n$ is appropriate follows from results in the sequel.

Given non-negative rational numbers $v_{1}, \ldots, v_{n}$ with sum 1 and an integer $m$ which is a multiple of the smallest common divisor of $v_{1}, \ldots, v_{n}$. Then

$$
s(n, v, k, u)=\max \frac{T(P, k, u)}{m},
$$

where this maximum is taken over all $m \times n 0,1$-matrices $P$ where the number of rows with exactly $i 1: \mathrm{s}$ is $m v_{i}$. 
The most general performance function is

$$
G(k, u, q)=\sup _{n} g(n, k, u, q)
$$

We conclude the preparations for the main result by introducing some notation relevant in this situation.

We call a matrix $P$ complete if all possible rows, that is, if all $\left(\begin{array}{l}n \\ q\end{array}\right)$ permutations of the $q 1$ :s, occur equally frequently as rows of $P$. The number of rows is thus necessarily divisible by $\left(\begin{array}{l}n \\ q\end{array}\right)$.

We also need the following three combinatorial functions. Let $I$ be a finite sequence of non-negative integers. Then we define:

$b(I)=$ the number of distinct integers in $I$

$a(I, j)=$ the number of occurences of the $j$ :th distinct integer in $I$, enumerated in size order, $1 \leq j \leq b(I)$.

$\pi(k, w, q, l)=$ the number of permutations of $q 1$ :s distributed in $k w$ slots, which are divided in $k$ sets with $w$ slots in each, such that the set with maximum number of 1 :s has exactly $l 1: \mathrm{s}$. 


\section{Process dependent performance functions}

We can now state the formula for $g(n, k, u, q)$, from which explicit formulas for the functions $s(n, v, u, q)$ and $G(k, u, q)$ follow.

THEOREM 6.1 Given positive integers $m, n, k, q$ and $u ; n \geq k$ and $n \geq q$.

In the case where $w=n / k$ is an integer, we have for all matrices $P$ of $m, n, q$-type:

$$
T(P, k, u) / m \leq g(n, k, u, q)=\frac{1}{u\left(\begin{array}{l}
n \\
q
\end{array}\right)} \sum_{l=1}^{\min (w, q)} \max (l, u) \pi(k, w, q, l) .
$$

If $w=n / k$ is not an integer, we let $w=\lfloor n / k\rfloor$ and denote the remainder of $n$ divided by $k$ by $n_{k}$, i.e. $n_{k}=n-k\lfloor n / k\rfloor$. Then we have for all matrices $P$ of $m, n, k$-type:

$$
\begin{gathered}
T(P, k, u) / m \leq g(n, k, u, q)= \\
\frac{1}{u\left(\begin{array}{c}
n \\
q
\end{array}\right)} \sum_{l_{1}=\max \left(0,\left\lceil\frac{q-\left(k-n_{k}\right) w}{n_{k}}\right\rceil\right)}^{\min (w+1, q)} \sum_{l_{2}=\max \left(0,\left\lceil\frac{q-l_{1} n_{k}}{k-n_{k}}\right\rceil\right)}^{\min \left(w, q-l_{1}\right)} \max \left(l_{1}, l_{2}, u\right) \times \\
\sum_{i=\max \left(l_{1}, q-l_{2}\left(k-n_{k}\right)\right)}^{\min \left(l_{1} n_{k}, q-l_{2}\right)} \pi\left(n_{k}, w+1, i, l_{1}\right) \pi\left(k-n_{k}, w, q-i, l_{2}\right) .
\end{gathered}
$$

In the special cases $\min (q, w)<l$ and $q>k l, \pi(k, w, q, l)=0$. Otherwise if $k=1$, $\pi(1, w, q, l)=\left(\begin{array}{l}n \\ l\end{array}\right)$. In all other cases we have

$$
\pi(k, w, q, l)=\left(\begin{array}{c}
w \\
l
\end{array}\right) \sum_{I}\left(\begin{array}{c}
w \\
i_{1}
\end{array}\right) \cdot \ldots \cdot\left(\begin{array}{c}
w \\
i_{k-1}
\end{array}\right) \frac{k !}{\Pi_{j=1}^{b\left(\left\{l, i_{1}, \ldots, i_{k-1}\right\}\right)} a\left(\left\{l, i_{1}, \ldots, i_{k-1}\right\}, j\right) !} .
$$

Here the sum is taken over all sequences of non-negative integers $I=\left\{i_{1}, \ldots, i_{k-1}\right\}$ which are decreasing: $i_{j} \geq i_{j+1}$ for all $j=1, \ldots, k-2$, bounded by $l: i_{1} \leq l$, and have sum $q-l: \sum_{j=1}^{k-1} i_{j}=q-l$.

For each $m, n, q$-type matrix $P$ the minimum

$$
T(P)=\min _{\text {all partitions } A} T_{A}(P)
$$

is attained for a partition where the sizes of the sets in the partition differ as little as possible.

The bound is optimal in the sense that if $\left(\begin{array}{l}n \\ q\end{array}\right)$ divides $m$, in which case there exist complete matrices, we have $T(P) / m=g(n, k, u, q)$ for all complete matrices $P$.

As described earlier $g(n, k, u, q)$ is defined also in the cases $k>n$ and $q>n$, here we have $g(n, k, u, q)=1$ and $g(n, k, u, q)=g(n, k, u, n)=\max \left(\left\lceil\frac{n}{k}\right\rceil / u, 1\right)$, respectively. A consequence of this and of the theorem is that for $k=1$ we have $g(n, 1, u, q)=$ $\max (\min (n, q) / u, 1)$. 
The 256 first values of $g(n, k, u, q)$, for $1 \leq n, k, u, q \leq 4$, are presented in graphics part 2. Part 3 of the graphics section show three plots of $g$ representing three cases of increasing degrees of cluster organization using the same number of processors.

The last factor in the function $\pi$ is obviously a multinomial cofficient, we have preferred to write it out.

The following lemma provides an algorithm which generates all decreasing sequences. It is proved in [2].

We say that the least decreasing sequence of length $\mu$ and sum $\sigma$ is the sequence $\left\{\left\lceil\frac{\sigma}{\mu}\right\rceil, \ldots,\left\lceil\frac{\sigma}{\mu}\right\rceil,\left\lfloor\frac{\sigma}{\mu}\right\rfloor, \ldots,\left\lfloor\frac{\sigma}{\mu}\right\rfloor\right\}$. If $\sigma_{\mu}$ is the remainder when $\sigma$ is divided by $\mu$, the number of $\left\lceil\frac{\sigma}{\mu}\right\rceil: \mathrm{s}$ is $\sigma_{\mu}$, and the number of $\left\lfloor\frac{\sigma}{\mu}\right\rfloor \mathrm{s}$ is $\mu-\sigma_{\mu}$, making the sum of the sequence $\sigma$.

LEMMA 6.2 Let $\lambda$ and $\sigma$ be non-negative integers and $\mu$ be a positive integer such that $\lambda \leq \sigma \leq \lambda \mu$.

Every sequence of $\mu$ integers in the interval $0 \leq i \leq \lambda$ which is decreasing, bounded by $\lambda$ and has sum $\sigma$ is generated exactly once by the following algorithm:

1. Take $I$ as the least decreasing sequence of length $\mu$ and sum $\sigma$.

2. Find the rightmost position in I, say $j_{1}$, which fullfills:

(a) $i_{j_{1}}<l$

(b) $i_{j_{1}}<i_{j_{1}-1}$ or $j_{1}=1$

(c) $i_{j_{1}+1}>0$

The algorithm terminates if no such $j_{1}$ can be found.

3. The next sequence is obtained from $I$ by increasing the entry in position $j_{1}$ by one, and replacing the subsequence $\left\{i_{j_{1}+1}, \ldots, i_{\mu}\right\}$ with the least decreasing subsequence of length $\mu-j_{1}$ and sum $\sum_{j=j_{1}+1}^{\mu} i_{j}-1$.

4. Go to step 2.

Observe that $\sum_{l=1}^{\min (w, q)} \pi(k, w, q, l)=\left(\begin{array}{c}n \\ q\end{array}\right)$ in the case $n / k$ an integer, and otherwise 


$$
\sum_{l_{1}=0}^{\min (w+1, q)} \sum_{l_{2}=0}^{\min \left(w, q-l_{1}\right)} \sum_{i=\max \left(l_{1}, q-l_{2}\left(k-n_{k}\right)\right)}^{\min \left(l_{1} n_{k}, q-l_{2}\right)} \pi\left(n_{k}, w+1, i, l_{1}\right) \pi\left(k-n_{k}, w, q-i, l_{2}\right)=\left(\begin{array}{l}
n \\
q
\end{array}\right),
$$

since we are only counting all permutations of the $q 1$ :s in the $n$ slots in different ways. Thus the formula can be regarded as a weighted average of the numbers $\{\max (l / u, 1): l=$ $1,2, \ldots, \min (\lceil n / k\rceil, q)\}$. In the case $u \geq \min (\lceil n / k\rceil, q)$ all these numbers are 1 , hence we in this case obtain $g(n, k, u, q)=1$. In multiprocessor terms this corresponds to a collapse of cluster allocation into dynamic allocation since then one cluster alone is equally large as the first multiprocessor.

Proof of the theorem: This proof is similar to the corresponding proof in [2] - we refer to that proof.

We next will establish a formula for the function $s$ in terms of $g$. Given non-negative rational numbers $v_{1}, \ldots, v_{n}$ with sum 1 and an integer $m$ which is a multiple of the smallest common divisor. Then $s$ has been defined as

$$
s(n, v, k, u)=\max \frac{T(P, k, u)}{m},
$$

where the maximum is taken over all $m \times n 0,1$-matrices $P$ which has $m v_{i}$ rows with $i 1$ :s.

\section{THEOREM 6.3}

$$
s(n, v, k, u)=\sum_{i=1}^{n} v_{i} g(n, k, u, i)
$$

Proof: As in the previous proof, given in [2], the argument of permuting the columns is performed. This gives a matrix $\tilde{P}$ of $n ! m$ rows, where $n ! x_{i}$ rows has exactly $i 1$ :s. Among the rows with $i$ 1:s all $\left(\begin{array}{c}n \\ i\end{array}\right)$ permutations occurs equally often. Denote the matix composed by these rows by $P_{i}$. Then Theorem 6.1 gives $T\left(P_{i}\right)=g(n, k, u, i) n ! x_{i}$. We obtain

$$
\begin{gathered}
s(n, v, k, u)=\max \frac{T(P, k, u)}{m}=\max \frac{T(\tilde{P}, k, u)}{n ! m} \\
=\frac{g(n, k, u, 1) n ! x_{1}+\ldots+g(n, k, u, n) n ! x_{n}}{n ! m}=\sum_{i=1}^{n} v_{i} g(n, k, u, i) .
\end{gathered}
$$

THEOREM 6.4 The function $g(n, k, u, q)$ has the following properties:

1. $g(n, k, u, q)$ is increasing in the variables $n$ and $q$, and decreasing in the variables $u$ and $k$.

2. For any positive integer $w, g(w k, k, u, q)$ and $g(w k, k, 1, k)$ are increasing as functions of $k$. 
3. Given positive integers $\nu, w \geq u$ and a positive real number $\alpha$, we have:

$$
\begin{gathered}
\max \left(\frac{w+1}{u}, 1\right)-\frac{1}{u}\left(1-\left(\frac{w}{\alpha}\right)^{-(w+1)}\right)^{\nu} \\
\geq \lim _{k \rightarrow \infty} g(w k+\nu, k, u, \alpha k) \\
\geq \max \left(\frac{w}{u}, 1, \max \left(\frac{w+1}{u}, 1\right)-\left(1-\left(\frac{w}{\alpha}\right)^{-(w+1)}\right)^{\nu}\right) .
\end{gathered}
$$

4. The function $g(n, k, u, q)$ is unbounded.

Note further that $g(n, k, u, q)=1$ if $u \geq \min (\lceil n / k\rceil, q)$ and if $k \geq n$, and $g(n, k, u, q)=$ $g(n, k, u, n)=\max (k / u, 1)$ for all $q \geq n$.

The upper and lower bounds of the functions $D(w, \nu)=\lim _{k \rightarrow \infty} g(w k+\nu, k, 1, k)$ formulated in the theorem are plotted in part 6 of the graphics section for $\nu=2, \ldots 6$.

Proof: (1): $g(n, k, u, q)=\min _{\text {all partitions } A} T_{A}(P) / m$, where $P$ is a complete $m, n, q$ type matrix. Let $A$ be a partition where the sizes of the sets differ as little as possible.

That $g$ is increasing as a function of $n$ follows by adding to the matrix $P$ one column of zeros only. We then obtain the non-complete $m, n+1, q$-type matrix $P_{0}$. This column is added to the partition $A$, producing $A_{0}$, in such a way that $A_{0}$ also has sizes of the sets which differ as little as possible. By the proof of theorem 2 it follows that $g(n, k, u, q)=$ $T_{A}(P) / m=T_{A_{0}}\left(P_{0}\right) / m \leq T_{A_{0}}\left(\tilde{P}_{0}\right) / m=g(n+1, k, u, q)$, where $\tilde{P}_{0}$ is a complete matrix of $m, n+1, q$-type. We can choose $m$ so that it is divisible by both $\left(\begin{array}{c}n \\ q\end{array}\right)$ and $\left(\begin{array}{c}n+1 \\ q\end{array}\right)$.

Consider $q<n$. If, at each row of $P$, the first 0 is replaced by 1 , we obtain the matrix $P_{1}$. Clearly we get $g(n, k, u, q)=T_{A}(P) / m \leq T_{A}\left(P_{1}\right) / m \leq T_{A}\left(\tilde{P}_{1}\right) / m=g(n, k, u, q+1)$, where $\tilde{P}_{1}$ is a complete matrix of the same type as $P_{1}$. Here we choose $m$ so that it is divisible by both $\left(\begin{array}{c}n \\ q\end{array}\right)$ and $\left(\begin{array}{c}n \\ q+1\end{array}\right)$.

Since $\max (1, l / u)$ is decreasing as a function of $u$, so is $g(n, k, u, q)$.

By adding an empty set to the partition $A$ we obtain the partition $A^{\prime}$. $A^{\prime}$ is usually not a partition where the sizes of the sets differ as little as possible. Thus, if $\tilde{A}^{\prime}$ is a partition with minimal difference in sizes and with the same number of sets as $A^{\prime}$, we have $g(n, k, u, q)=T_{A}(P) / m=T_{A^{\prime}}(P) / m \geq T_{\tilde{A}^{\prime}}(P) / m=g(n, k+1, u, q)$.

(2): By adding one column of 1 :s and $w-1$ columns of 0 :s to the matrix $P$, the increasing properties in (2) follow with an argument similar to those in the proof of (1).

The idea of the proof of (3) is to exploit the fact that $g$ is a weighted average of the numbers $\{\max (l / u, 1): l=1,2, \ldots, \min (\lceil n / k\rceil, q)\}$. We calculate the limit of the weight $W$ for the number $\max ((w+1) / u, 1)$ in the weighted average as $k \rightarrow \infty$. Then the estimate follows from $W \max ((w+1) / u, 1)+(1-W) 1 \leq \lim _{k \rightarrow \infty} g(w k+\nu, k, u, \alpha k) \leq$ $W \max ((w+1) / u, 1)+(1-W) \max (w / u, 1)$. The proof is given in [2], the only difference here is that the number $\alpha$ enters the proof.

(4) follows immediately from (3).

Analogously to the function $g(n, k)$ described in [2], the existence of plateaus for the graph of $g(n, k, u, q)$ follows from these results. For $w+1 \geq w$ and taking $\nu$ large enough 
we get

$$
\left|\lim _{k \rightarrow \infty} g(w k+\nu, k, u, \alpha k)-\frac{w+1}{u}\right| \leq \epsilon_{\nu},
$$

where $\epsilon_{\nu}$ is small. Thus there exists one plateau at levels $w / u$ for each integer $w$, i.e. for each $\epsilon>0$ there is a domain $\left\{(n, k): n>w k, n<(w+1) k+\nu_{w}, k>\mu_{w}\right\}$ so that $|g(n, k, u, \alpha k)-w / u|<\epsilon$ here. $\nu_{w}$ and $\mu_{w}$ appear to increase very rapidly with $w$.

The function $g(n, k)=g(n, k, 1, k)$ which compares static with dynamic allocation is neither increasing nor decreasing as a function of $k$ (see [2]). A plot of the local extreme values of $g(n, k)$ as a function of $k$, for each fixed $n$, is shown in graphics part 8. Detail structure otherwise not visible is revealed in this plot.

The optimal performance function $g(n, k)$ thus has a shape resembling a winding staircase with constant step height and an infinite number of steps, where each step is narrower, less sharp-edged and much more distant from the origin than the previous step.

\section{The process independent performance function}

It turns out that it also is possible to compute explicitely the optimal performance function $G(k, u, q)$ :

THEOREM 7.1 For any positive integers $k, u$ and $q$,

$$
G(k, u, q)=\lim _{n \rightarrow \infty} g(n, k, u, q)=\sup _{n \in \mathbf{N}} g(n, k, u, q),
$$

where $G(1, u, q)=\max (q / u, 1)$, and if $k>1$ :

$$
G(k, u, q)=\frac{k ! q !}{k^{q} u} \sum_{l=1}^{l=q} \frac{\max (l, u)}{l !} \sum_{I}\left(\Pi_{j=1}^{k-1} i_{j} ! \Pi_{j=1}^{b(\{l\}+I)} a(\{l\}+I, j) !\right)^{-1} .
$$

The sum is taken over all sequences $I=\left\{i_{1}, \ldots, i_{k-1}\right\}$ of non-negative integers which are decreasing; $i_{j} \geq i_{j+1}$ for all $j=1, \ldots, k-2$, bounded by $l ; i_{1} \leq l$, and have sum $q-l$; $\sum_{j=1}^{k-1} i_{j}=q-l$.

Further, the function $G(k, u, q)$ is decreasing as a function of $k$ and as a function of $u$, and increasing as a function of $q . G(k)=G(k, 1, k)$ is an increasing function.

Here we use the notation $\{l\}+I=\left\{l, i_{1}, \ldots, i_{k-1}\right\}$.

In part 1 of the graphics section level curves of the function $G(k, u, q)$ are depicted. Part 4 and 5 show two ways in which the function $G$ can be used to compare the performance of allocation strategies.

In the case of static allocation and $k=q$, the case considered in [2], we get $G(k)=$ $G(k, 1, k)$, where

$$
G(k)=\frac{(k !)^{2}}{k^{k}} \sum_{l=1}^{l=k} \frac{1}{(l-1) !} \sum_{I}\left(\Pi_{j=1}^{k-1} i_{j} ! \Pi_{j=1}^{b(\{l\}+I)} a(\{l\}+I, j) !\right)^{-1} .
$$

The last sum is taken over the same sequences $I$ as in the previous formula, with $q=k$. 
Note that the number of terms in the sum increases rapidly with $k$. However, being unit fractions with products of factorials as denominators, all terms are very small if $k$ is large. From the Stirling formula it indeed follows that $(k !)^{2} \gg k^{k} \gg k$ ! for large $k$.

We give the first values of $G(k)$ as maximally reduced rational numbers, prime factorizations and decimal expansions:

$$
\begin{aligned}
& G(1)=1 \\
& G(2)=\frac{3}{2}=1.5 \\
& G(3)=\frac{17}{9}=\frac{17}{3^{2}}=1.888 \ldots \\
& G(4)=\frac{17}{8}=\frac{17}{2^{3}}=2.125 \\
& G(5)=\frac{1429}{625}=\frac{1429}{5^{4}}=2.2864 \\
& G(6)=\frac{3121}{1296}=\frac{3121}{2^{4} 3^{4}}=2.40818 \ldots \\
& G(7)=\frac{295189}{117649}=\frac{211 \cdot 1399}{7^{6}}=2.50907 \ldots \\
& G(8)=\frac{680849}{262144}=\frac{13 \cdot 83 \cdot 631}{2^{18}}=2.597232 \ldots \\
& G(9)=\frac{38404547}{14348907}=\frac{43 \cdot 107 \cdot 491 \cdot 1717}{3^{15}}=2.67648 \ldots \\
& G(10)=\frac{274868911}{100000000}=\frac{3929 \cdot 69959}{2^{8} 5^{8}}=2.74868911 .
\end{aligned}
$$

In part 7 of the graphics section the function $G(k)$ and a quantitative plot of the limiting procedure $\lim _{n \rightarrow \infty} g(n, k)=G(k)$ are shown.

Proof: By Theorem 6.4 the function $g(n, k, u, q)$ is increasing as a function of $n$, hence the limit and the supremum in the theorem coincide. Further it follows that $\lim _{n \rightarrow \infty} g(n, k, u, q)=\lim _{w \rightarrow \infty} g(w k, k, u, q)$. Note also that exactly the same decreasing sequences are generated in the function $\pi(k, w, k, l)$ for all $w \geq k$, if $l$ and $k$ are fixed. This fact makes it possible to explicitly compute the function $G(k, u, q)$. When letting $w \rightarrow \infty$, it is enough to study the behaviour of the $w$-dependent part of each term, which is

$$
\frac{\left(\begin{array}{c}
w \\
l
\end{array}\right)\left(\begin{array}{c}
w \\
i_{1}
\end{array}\right) \cdot \ldots \cdot\left(\begin{array}{c}
w \\
i_{c}
\end{array}\right)}{\left(\begin{array}{c}
w k \\
q
\end{array}\right)} .
$$

We will next prove that

$$
\lim _{w \rightarrow \infty} \frac{\left(\begin{array}{c}
w \\
l
\end{array}\right)\left(\begin{array}{c}
w \\
i_{1}
\end{array}\right) \cdot \ldots \cdot\left(\begin{array}{c}
w \\
i_{c}
\end{array}\right)}{\left(\begin{array}{c}
w k \\
q
\end{array}\right)}=\frac{q ! k^{-q}}{l ! i_{1} ! \cdot \ldots \cdot i_{c} !} .
$$

Here $c$ is the number of nonzero entries in the sequence $I$. In the following we will use the fact that $l+i_{1}+\ldots+i_{c}=q$. From the Stirling formula $n ! \approx\left(\frac{n}{e}\right)^{n} \sqrt{2 \pi n}$, we get for large $n$ :

$$
\left(\begin{array}{l}
n \\
k
\end{array}\right) \approx \frac{e^{-k}}{k !} \sqrt{\frac{n}{n-k}} \frac{n^{n}}{(n-k)^{n-k}}
$$


Thus the quotient approximately equals

$$
\begin{gathered}
\frac{e^{-l}}{l !} \sqrt{\frac{w}{w-l}} \frac{w^{w}}{(w-l)^{w-l}} \frac{e^{-i_{1}}}{i_{1} !} \sqrt{\frac{w}{w-i_{1}}} \frac{w^{w}}{\left(w-i_{1}\right)^{w-i_{1}}} \cdot \ldots \\
\cdot \frac{e^{-i_{c}}}{i_{c} !} \sqrt{\frac{w}{w-i_{c}}} \frac{w^{w}}{\left(w-i_{c}\right)^{w-i_{c}}} \frac{q !}{e^{-q}} \sqrt{\frac{k w-q}{k w}} \frac{(k w-q)^{k w-q}}{(k w)^{k w}}= \\
\frac{q !}{l ! i_{1} ! \cdot \ldots \cdot i_{c} !} \\
\left(1-\frac{q}{k w}\right)^{k w} \frac{w}{(w-l)^{w-l}\left(w-i_{1}\right)^{w-i_{1}} \cdot \ldots \cdot\left(w-i_{c}\right)^{w-i_{c}}}(k w-q)^{-q} .
\end{gathered}
$$

The first line after the last equality clearly is independent of $w$. The second line tends to 1. The first factor of the third line tends to $e^{-q}$. The second factor can be written as

$$
\left(1+\frac{l}{w-l}\right)^{w}\left(1+\frac{i_{1}}{w-i_{1}}\right)^{w} \cdot \ldots \cdot\left(1+\frac{i_{c}}{w-i_{c}}\right)^{w} .
$$

Now,

$$
\left(1+\frac{i}{w-i}\right)^{w} \rightarrow e^{i} \text { as } w \rightarrow \infty
$$

so we get

$$
e^{l} e^{i_{1}} \cdot \ldots \cdot e^{i_{c}}=e^{q}
$$

as $w \rightarrow \infty$, cancelling the factor $e^{-q}$. From the third factor we get

$$
\left(\frac{k w-q}{w-l}\right)^{-l}\left(\frac{k w-q}{w-i_{1}}\right)^{-i_{1}} \cdot \ldots \cdot\left(\frac{k w-q}{w-i_{c}}\right)^{-i_{c}} \rightarrow k^{-l} k^{-i_{1}} \cdot \ldots \cdot k^{-i_{c}}=k^{-q} .
$$

Thus the third line above contributes with a factor $k^{-q}$ as $w \rightarrow \infty$.

Since $g(n, 1, u, q)=\max (\min (n, q) / u, 1)$, it immediately follows that $G(1, u, q)=$ $\max (q / u, 1)$. Note that this case represents the worst case ratio of dynamic versus dynamic allocation, with $u$ and $q$ processors respectively. This completes the proof of the formula for $G(k, u, q)$.

The increasing properties for $G(k, u, q)$ and $G(k)$ follow from (3) and (4) of theorem 6.4. For example, the inequality $g(w k, k, u, q) \leq g(w(k+1), k+1, u, q)$ transforms into the inequality $G(k, u, q) \leq G(k+1, u, q)$ as $w \rightarrow \infty$.

We conclude with some notes on the computability of the functions.

The variable $u$ does not affect the number of floating point operations necessary for computing the value of the function $g(n, k, u, q)$. This number increases slightly with $q$ and $n$, if $n$ is a multiple of $k$, because of the larger values in the binomials and factorials to be computed. However, the number of operations increases rapidly with the variable $k$ since the number of decreasing sequences increase rapidly. This is also true for the 
variable $n$ if it is not a multiple of $k$. The function $h(n, k, u, q)=g(k\lceil n / k\rceil, k, u, q)$ is thus essentially faster to compute, since only a fraction of the values are needed, and those which are used are the computationally fastest. Part 9 of the graphics section shows $g(n, k), h(n, k)=h(k\lceil n / k\rceil, k, u, q)$ and the difference $h(n, k)-g(n, k)$ for $n, k \leq 50$. Since $g(n, k)$ is increasing as a function of $n$ we obviously have $h(n, k) \geq g(n, k)$, with equality at the straight lines $n=w k$ for any positive integer $w$.

\section{References:}

[1] R. L. Graham, Bounds on Multiprocessing Anomalies, SIAM Journal of Applied Mathematics, Vol. 17, No 2, March 1969, pp. 416-429.

[2] H. Lennerstad, L. Lundberg, An Optimal Execution Time Estimate of Static versus Dynamic Allocation in Multiprocessor Systems, SIAM Jou. of Comp., Vol. 24, No. 4, pp.751-764, August 1995.

[3] H. Lennerstad, L. Lundberg, Optimal Scheduling Results for Parallel Computing, SIAM News, Vol. 27, No. 7, 1994. In Applications of Advanced Architecture Computers, ed. Greg Astfalk, SIAM, 1996.

[4] H. Lennerstad, L. Lundberg, Combinatorics for Multiprocessor Scheduling Optimizationand Other Contexts in Computer Architecture, Proceedings of the Conference of Combinatorics and Computer Science, Brest, France, 1995. In Lecture Notes of Computer Science 1120, Springer Verlag, 1996.

[5] H. Lennerstad, L. Lundberg, Combinatorial Formulas for Optimal Cache Memory Efficiency, SIAM News, 1996.

[6] L. Lundberg, H. Lennerstad, An Optimal Bound on the Gain of Using Dynamic versus Static Allocation in Multiprocessor Computers, Technical Report, Univ. of Karlskrona/Ronneby (1992).

[7] L. Lundberg, H. Lennerstad, An Optimal Performance Bound on the Gain of Using Dynamic versus Static Allocation in Multiprocessors with Clusters, Technical Report, Univ. of Karlskrona/Ronneby (1993).

[8] L. Lundberg, H. Lennerstad, An Optimal Upper Bound on the Minimal Completion Time in Distributed Supercomputing, Proceedings of the 8th ACM Conference on Supercomputing, Manchester, England, July 1994.

[9] L. Lundberg, H. Lennerstad, An Optimal Lower Bound on the Maximal Speedup in Multiprocessors with Clusters, First International Conference on Algorithms and Architechtures for Parallel Processing, Australia, April 1995.

[10] L. Lundberg, H. Lennerstad, An optimal bound on the gain of using one large processor cluster instead of a number of small clusters, Proceedings of the ISCA International Conference on Parallel and Distributed Computing Systems, Orlando, Fl, 21-23 Sept 1995. 
[11] L. Lundberg, H. Lennerstad, Optimal worst case formulas comparing cache memory associativity, Research Report 5/95, Univ. of Karlskrona/Ronneby (1995).

[12] L. Lundberg, Static Process Allocation Using Information about Program Behavior, Proceedings of the 24'th Hawaii International Conference on System Sciences, Hawaii, pp. 1-9 (1991).

[13] C.C. Price, Task Allocation in Data Flow Multiprocessors: an annotated bibliography, Comput. Archit. News, vol. 19, no. 1, (March 1991) pp. 128-134.

[14] Proceedings of the Twentieth Southeastern Conference on Combinatorics, Graph theory and Computing, Boca Raton, FL (1989).

[15] Proceedings of the Twenty-second Southeastern Conference on Combinatorics, Graph theory and Computing, Baton Rouge, LA, (1991).

[16] B. Shirazi, Ming-fang Wang, Heuristic Functions for Static Task Allocation, Microprocess. Microprogr. (Netherlands) Vol 26, no. 3, (Oct 1989) pp. 187-194.

[17] J. Zahorjan, C. McCann, Processor Scheduling in Shared Memory Multiprocessors, Performance Evaluation Review (USA), vol. 18, no. 1, (May 1990). 